\section{DENTAL BLEACHING EDUCATION AND TRAINING}

The British Dental Bleaching Society (BDBS) provides training and education and is the only UK body to offer certification of tooth whitening procedures to all members of the dental team. The dental team can gain the required level of clinical and academic knowledge and skill necessary to provide safe and predictable results.

There are two levels of membership to the society, regular and gold, and once joined you will have access to the latest information on bleaching, education and training.

The Dental Directory sponsors the BDBS, supporting the society and members to ensure the correct practice of tooth whitening. Members are eligible for an exclusive package of benefits provided by The Dental Directory.

Reader response number 62

\title{
PRECISION IMAGERY OF TREATMENT SITE
}

The Kodak 1500 intraoral camera provided by PracticeWorks is an easy to use, lightweight device which allows precision imagery of the treatment site to be taken in either stills or video.

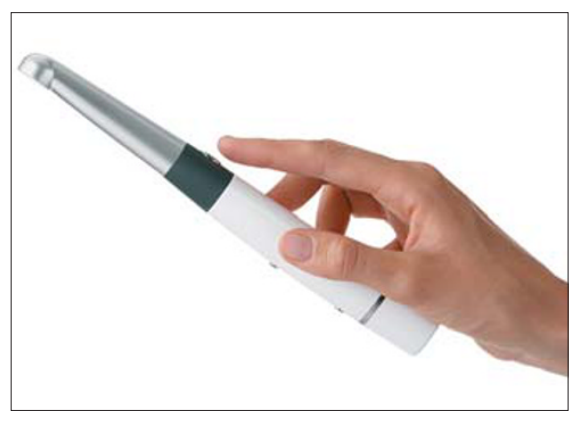

The revolutionary liquid lens technology focuses like the human eye, eliminating undesired light reflection and automatically adjusting to the light conditions. For improved handling, the device utilises wireless technology, meaning fewer cables in the surgery.

With a combined docking and charging station, this device is easily shared by multiple operators. Its auto-focus and high-resolution video or still image gives absolute clarity in view of the treatment site.

Reader response number 63

\section{LOCATING HIDDEN CANALS}

Dentsply Maillefer, together with Professor Berutti, Professor Cantatore and Dr Castellucci, have developed a range of five ultrasonic tips based on a simple concept, one tip - one clinical indication, to facilitate and optimise the preparation of the access cavities and localisation of the orifices of hidden canals.

The Start-X tip 1 is for the refining of the access cavity walls. The tip can be used to remove restorations and filling materials, caries and dentine interferences from the access cavity walls thus allowing direct access to the root canal orifices. The Start-X tip 2 will help to locate the second mesiobuccal canal of maxillary molars by removing the dentinal layer in the pulp chamber floor, between the mesiobuccal (MB1) and the

\section{SOFT BRISTLE AND}

\section{SINGLE-TUFT TOOTHBRUSHES}

The Curaprox range of soft bristle or single-tuft toothbrushes ensures teeth are effectively cleaned whilst keeping the gumline intact, an effective oral hygiene procedure especially when used in conjunction with interdental brushes.

Curaprox also has products specifically designed for the very young with the award winning CURAbaby teething ring. There is also a Denture and Gum Care Set for effective care and cleaning which helps to maintain the original colour of the denture's acrylic resin.

Reader response number 65

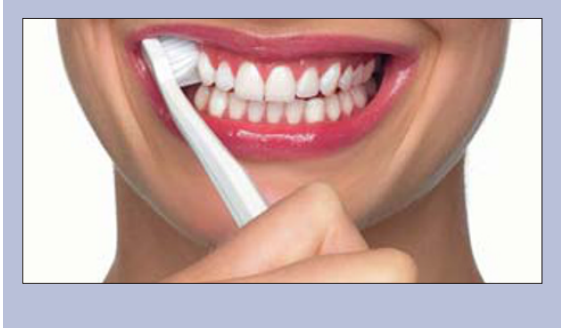

\section{HELP NEW PATIENTS FIND YOU}

Cosmetic dentistry website www.cosmeticdentistryguide.co.uk receives over 140,000 visitors a month to investigate treatments, technologies and also dental professionals near to them.

The site is ranked number one by Google for many search items including teeth straightening, cosmetic dentists, dental implants, crowns and bridges, teeth whitening, dental bridges, smile makeovers and veneers.

cosmeticdentistryguide.co.uk was built to be a quick and easy to use option for people exploring cosmetic dentistry treatments and offers a simple dialogue that patients understand. To make sure that you are seen by potential new clients and receive a large amount of online traffic, you can be listed in the 'Find a local cosmetic dentist' directory with a link directly to your practice.

For less than $£ 100$ a month patients in your area who are seeking cosmetic dentistry will be directed to your practice. The site also features news updates about the latest innovations in cosmetic dentistry.

\section{Reader response number 66}

